\title{
Extension of the SIESTA MHD equilibrium code to free-plasma-boundary problems
}

\author{
H. Peraza-Rodriguez, R. Sanchez, J.M. Reynolds-Barredo and V. Tribaldos \\ Departamento de Física, Universidad Carlos III de Madrid, 28911 Leganés, Madrid, Spain \\ J. Geiger \\ Max-Planck-Institut für Plasmaphysik, 17491 Greifswald, Germany \\ S.P. Hirshman, M. Cianciosa \\ Oak Ridge National Laboratory, Oak Ridge, 37831 TN, USA
}

(Dated: July 20, 2017)

\begin{abstract}
SIESTA [S.P. Hirshman, R. Sanchez and C.R. Cook, Phys. Plasmas 18, 062504 (2011)] is a recently developed MHD equilibrium code designed to perform fast and accurate calculations of ideal MHD equilibria for three-dimensional magnetic configurations. Since SIESTA does not assume closed magnetic surfaces, the solution can exhibit magnetic islands and stochastic regions. In its original implementation SIESTA addressed only fixed-boundary problems. That is, the shape of the plasma edge, assumed to be a magnetic surface, was kept fixed as the solution iteratively converges to equilibrium. This condition somewhat restricts the possible applications of SIESTA. In this paper we discuss an extension that will enable SIESTA to address free-plasma-boundary problems, opening up the possibility of investigating problems in which the plasma boundary is perturbed either externally or internally. As an illustration, SIESTA is applied to a configuration of the W7-X stellarator.
\end{abstract}

PACS numbers: 52.25.Fi, 52.35.Ra, 52.25.Gj, 05.40.-a

\section{INTRODUCTION}

SIESTA is an iterative MHD equilibrium solver that looks for lower energy states starting from nearby equilibria with nested magnetic surfaces ${ }^{1}$, being one of just a handful of MHD equilibrium $\operatorname{codes}^{2-4}$ that does not assume the existence of magnetic surfaces. SIESTA uses the nested equilibrium solution found by the VMEC $\operatorname{code}^{5}$ to provide: 1) a (fixed) background set of quasipolar coordinates, $\left(s_{v}, \theta_{v}\right.$ and $\left.\phi_{v}\right)$, in which calculations are carried out, and 2) an initial guess for the equilibrium magnetic field and pressure fields to start the iterative search of an equilibrium solution. Here, $\phi_{v}=\phi$ is the geometrical toroidal angle; $\theta_{v}=\theta^{*}$, the non-geometrical poloidal angle that VMEC internally uses to maximize the compression of the harmonic content of the solution ${ }^{6}$; finally, $s_{v}=\sqrt{s}$, with $s$ being the normalized magnetic toroidal flux that VMEC uses as radial coordinate.

SIESTA, as VMEC, is a spectral code in which the curvilinear components of all fields are expanded in Fourier series in the poloidal $\left(\theta_{v}\right)$ and toroidal $\left(\phi_{v}\right)$ angles. For the sake of simplicity, SIESTA currently assumes stellarator symmetry ${ }^{7}$, so that all fields exhibit either cosine,

$$
C\left(s_{v}, \theta_{v}, \phi_{v}\right)=\sum_{m=0}^{M} \sum_{n=-N}^{N} C_{m n}\left(s_{v}\right) \cos \left(m \theta_{v}+n N_{p} \phi_{v}\right)
$$

or sine,

$$
S\left(s_{v}, \theta_{v}, \phi_{v}\right)=\sum_{m=0}^{M} \sum_{n=-N}^{N} S_{m n}\left(s_{v}\right) \sin \left(m \theta_{v}+n N_{p} \phi_{v}\right),
$$

symmetry, depending on the parity of the field of interest. The values of the harmonics of the three contravariant components of the magnetic field (i.e., $B^{s_{v}}$ [sine parity], $B^{\theta_{v}}$ [cosine] and $B^{\phi_{v}}$ [cosine] $)$ and the plasma pressure $(p$, cosine) are iteratively varied by SIESTA until it reaches a minimum of the total MHD energy integrated over the plasma volume,

$$
W=\int\left[\frac{B^{2}}{2 \mu_{0}}+\frac{p}{\gamma-1}\right] d V
$$

consistent with the imposed boundary conditions and MHD conservation laws for flux and mass. As it is well known, the MHD energy becomes quasi-stationary only when the ideal MHD force, $\mathbf{J} \times \mathbf{B}-\nabla p$, vanishes ${ }^{8}$. SIESTA looks for zeros of this nonlinear force, in terms of the plasma displacement vector, using an iterative nonlinear Newton method ${ }^{1}$. The use of the VMEC coordinate system ensures that the number of Fourier harmonics that need to be included in SIESTA is kept down to a minimum, improving considerably both the convergence of the Newton method and the overall performance of the code. In addition, the solution of the linear problem that appears at each step of the non-linear Newton method is also searched iteratively, combining Krylov and conjugate-gradient methods with accurate physicsbased preconditioning to further accelerate convergence to the desired tolerance ${ }^{1}$.

Another subtle aspect of SIESTA is that it does allow for a certain (and controlled) departure from ideal MHD during the iterative procedure. In purely ideal MHD codes with nested surfaces, magnetic islands (and the stochastic regions that may result from their superposition) are prevented from opening up by the formation of (parallel) current sheets at the resonant mag- 
netic surfaces ${ }^{9-11}$. SIESTA allows for the diffusion of these resonant currents (and therefore, the violation of the frozen-flux theorem of ideal MHD) by interlacing the preconditioned ideal steps with a few resistive steps. In real devices, the very small diffusivity makes this a very slow physical process, that is significant only at the current sheet region. SIESTA accelerates this process by artificially increasing the resistivity (and also, via the rescaling of the relevant eigenvalues done by the internal preconditioning), but its application during the iterative procedure is done in a way that the main (non-resonant) components are barely affected [More details about the use of resistivity can be found in the original SIESTA reference ${ }^{1}$.].

As it was mentioned earlier, the original version of SIESTA was developed as a fixed-boundary code $^{1}$. That is, its spatial domain is the plasma volume, whose boundary is defined by the isosurface $s_{v}=1$. Boundary conditions are imposed at this boundary that ensure that, while the MHD energy is minimized, any plasma displacement considered always vanishes there. As a result, both the magnetic field and the plasma pressure remain fixed at the boundary during the iterative procedure, with $B^{s}$ (since $s_{v}=1$ is a magnetic surface) and $p$ set to zero. There are however situations of relevance in which one would like to be able to calculate the changes to the plasma boundary caused by different actions. For instance, during the application of resonant magnetic perturbations close to the plasma edge, as those often used to try to control ELM activity in tokamak $\mathrm{H}$ modes ${ }^{12}$. Or when unbalanced plasma currents appear in zero-current stellarators, driven for instance by external heating or the pressure gradient, as could be the case of the W7-X stellarator ${ }^{14}$. For that reason, this paper introduces a new methodology that enables SIESTA to perform free-plasma-boundary equilibrium calculations.

The paper is organized as follows. In Sec. II, the fundamentals of the new approach are described. They include the extension of the calculation domain beyond the plasma edge, the construction of an adequate numerical mesh throughout the extended region (Sec. II A) and the construction of a proper initial guess, for both magnetic field (Sec. II B) and pressure (Sec. IIC), over the extended domain. The extended capabilities of the new version of SIESTA are illustrated in Sec. III, where we use it on a selected configuration of the W7-X stellarator. Then, some final conclusions will be drawn in Sec. IV.

\section{FREE-PLASMA-BOUNDARY EXTENSION PROCEDURE}

In order to enable SIESTA to perturb the plasma boundary while looking for equilibrium solutions with lower MHD energy, the computational domain needs to be expanded so that the plasma boundary (the old $s_{v}=1$ ) becomes an internal surface (that will probably cease to coincide with the $s_{v}=1$ isosurface of the fixed

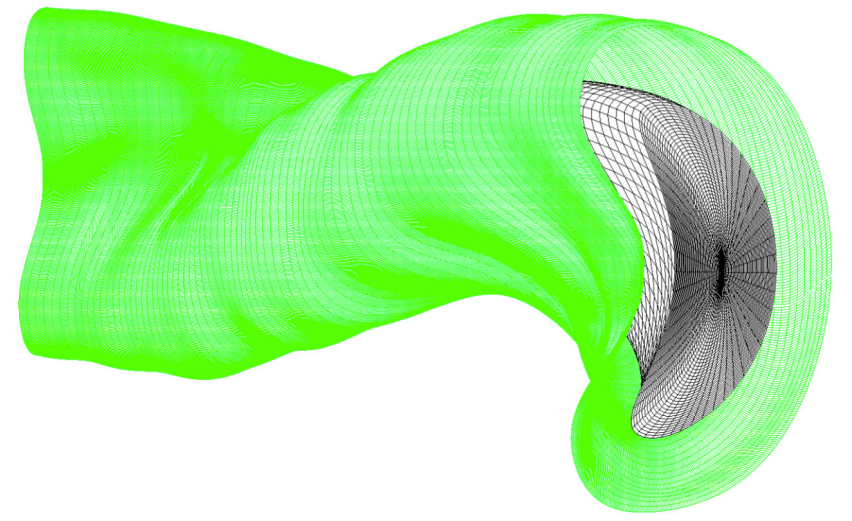

Figure 1. Illustration of the extended domain used by SIESTA for the W7-X stellarator runs that will be discussed in Sec. III. (A smoothed version of) W7-X's vaccum vessel, shown in green, defines the $s_{v}=s_{w}$ surface. The magnetic surfaces of the VMEC solution for the same run are shown in black, the last of them corresponding to $s_{v}=1$.

background coordinate system). Although any volume that includes the plasma could be used, it seems natural to consider the volume inside the vacuum vessel of the device of interest (see Fig. 1). The strategy we will then follow is to consider the boundary of the extended volume as a new fixed boundary, at which suitable boundary conditions will be imposed. In this way, SIESTA can be run in fixed boundary mode with respect to the extended volume, while being run in free boundary mode with respect to the plasma edge. Several things must be done before SIESTA can be run in this manner, though. First, the background coordinate system that VMEC provides must be extended over the region going from the original plasma boundary $\left(s_{v}=1\right)$ to the new boundary. Secondly, suitable initial guesses for both the magnetic and pressure fields, from which SIESTA will start to iterate, must also be provided over the extended region.

\section{A. Mesh extension}

The extension of SIESTA's background coordinate system beyond the plasma edge of the VMEC solution (i.e., $s_{v}=1$ ) is done as follows. First, it is assumed that the vacuum vessel (or any other external surface chosen for this purpose) corresponds to an isosurface of the extended background coordinate system $s_{v}=s_{w}$, for some value $s_{w}>1$ yet to be determined. Although it should be kept in mind that $s_{v}=s_{w}$ needs not be a magnetic surface. In fact, since magnetic surfaces typically vary during SIESTA's iterative procedure, be it in fixed- or free-plasma-boundary mode, there is no guarantee that $s_{v}=s_{0}$ will remain a magnetic surface in the final equilibrium solution for any value of $s_{0}$, except for $s_{0}=1$ in fixed-boundary mode. Then, $N_{\phi}$ toroidal planes are 


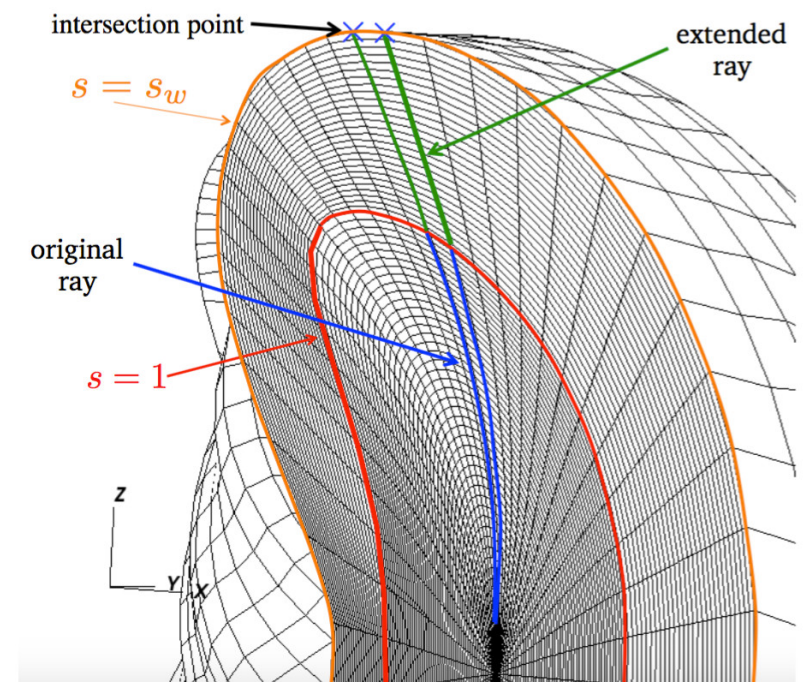

Figure 2. Sketch illustrating the construction of the extended coordinate system in the region in between the plasma and the vacuum vessel. First, poloidal rays are extended at each toroidal plane until they intersect the vessel. The collection of all intersections form the $s_{v}=s_{w}$ surface, where $s_{w}$ is to be determined. Intermediate isosurfaces are constructed for $s_{v}>1$ by dividing each poloidal ray in a fixed number of equal segments. The value of the label $s_{v}$ at each of the new surfaces is defined by requiring that the increase in volume roughly grows as the average minor radius.

considered for the angle values,

$$
\phi_{v}^{i}=\frac{2 \pi}{N_{p}}\left(\frac{i-1}{N_{\phi}}\right), \quad i=1, \cdots, N_{\phi},
$$

being $N_{p}$ the number of periods of the configuration. On each of these toroidal planes, $N_{\theta}$ poloidal angles are selected,

$$
\theta_{v}^{k}=2 \pi\left(\frac{k-1}{N_{\theta}}\right), \quad k=1, \cdots, N_{\theta} .
$$

At the $i$-th toroidal plane, one can easily trace the ray that goes from the axis $\left(s_{v}=0\right)$ to the plasma edge $\left(s_{v}=1\right)$, i.e. $\theta_{v}=\theta_{v}^{k}$ in real space, by summing up the series,

$$
\begin{aligned}
& R_{k, i}\left(s_{v}^{l}\right)=\sum_{m=0}^{M} \sum_{n=-N}^{N} R_{m n}\left(s_{v}^{l}\right) \cos \left(m \theta_{v}^{k}+n N_{p} \phi_{v}^{i}\right) \\
& Z_{k, i}\left(s_{v}^{l}\right)=\sum_{m=0}^{M} \sum_{n=-N}^{N} Z_{m n}\left(s_{v}^{l}\right) \sin \left(m \theta_{v}^{k}+n N_{p} \phi_{v}^{i}\right)
\end{aligned}
$$

for increasing $s_{v}^{l}=(l-1) d s, l=1,2,3 \cdots N_{s}$. Here, $d s=1 /\left(N_{s}-1\right)$ is the spacing in between isosurfaces that SIESTA uses for fixed-boundary calculations.

To extend the rays beyond $s_{v}=1$, we extrapolate both $R_{m n}\left(s_{v}^{l}\right)$ and $Z_{m n}\left(s_{v}^{l}\right)$ using a second-order polynomial fit of their values at $s_{v}^{N_{s}}, s_{v}^{N_{s}-1}$ and $s_{v}^{N_{s}-2}$ (see Fig. 2). Thus, when summing the series for $l>N_{s}$, the rays cross

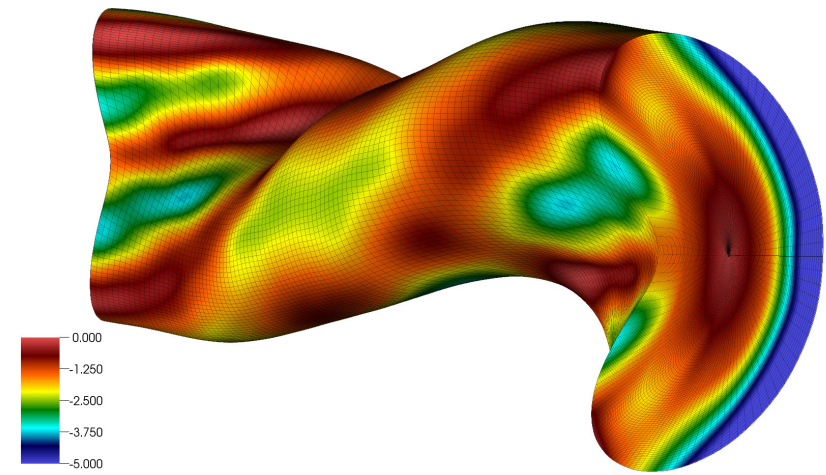

Figure 3. Contour plot of the jacobian, $\sqrt{g}$, over the extended computational volume built for the W7-X configuration examined in this paper by using the prescription described in the main text. Isosurfaces of constant $s_{v}$, rays of constant $\theta_{v}$ and toroidal planes can be clearly identified from the gridding.

the plasma edge and move towards the vacuum vessel. In general, each ray will require a different value of $l>$ $N_{s}$ (say, $l=l_{i k}$ for the $i$-th ray on the $k$-th toroidal plane) to cross the vacuum vessel. Therefore, there is no single value of $l$ such that $s_{v}=s_{v}^{l}$ provides a unique label for the vacuum vessel, but it is required that it be an isosurface of the extended background coordinate system. In order to define a more adequate label we proceed as follows. First, the last isosurface of the domain, $s_{v}=s_{w}$, is defined as the collection of the intersections with the vacuum vessel of all poloidal rays in all toroidal planes (in cases in which the shape of the last surface contains regions with large degrees of indentation, the intersection points obtained may lie too close to each other in some parts of the boundary; in those cases, an on-boundary resampling of the intersection positions is done before continuing the procedure). The actual value of $s_{w}$ still needs to be determined. Next, we divide the part of each ray that goes from $s_{v}=1$ to $s_{v}=s_{w}$ in $N_{s}^{\prime}$ equal segments. Isosurfaces in the extended volume are now successively defined by the collection of the end points of the first segment of all rays, the collection of the end points of the second segment of all rays, and so forth until the vacuum vessel is reached. The value of the label $s_{v}^{l}$ for the $l$-th collection $\left(l>N_{s}\right)$ is then assigned by requiring that the increment in volume enclosed by the $l$-th isosurface,

$$
\begin{aligned}
V\left(s_{v}^{l}\right) & =\int_{0}^{2 \pi} d \theta_{v} \int_{0}^{2 \pi} d \phi_{v} \sqrt{g}\left(s_{v}^{l}\right)= \\
& =\int_{0}^{2 \pi} d \theta_{v} \int_{0}^{2 \pi} d \phi_{v} R\left(\frac{\partial R}{\partial s_{v}} \frac{\partial Z}{\partial \theta_{v}}-\frac{\partial Z}{\partial s_{v}} \frac{\partial R}{\partial \theta_{v}}\right)
\end{aligned}
$$

grows as the square of the radial label $s_{v}$ that defines each magnetic surface. That is,

$$
s_{v}^{l}=s_{v}^{l-1}\left(\frac{V\left(s_{v}^{l}\right)}{V\left(s_{v}^{l-1}\right)}\right)^{1 / 2}, \quad l=N_{s}+1, \cdots, N_{s}+N_{s}^{\prime} .
$$


Once the new radial label is defined in this way, the jacobian $\sqrt{g}$ (see Fig. 3), as well as all other metric quantities, can be easily computed in the extended volume from the $R_{m n}$ and $Z_{m n}$ harmonics that define each isosurface for $s_{v}>1$. These harmonics are obtained in turn via the Fourier inversion of the $R_{k, i}\left(s_{v}^{l}\right), Z_{k, i}\left(s_{v}^{l}\right)$ pairs, for $l>N_{s}$ (see Eq. 7). As the final touch of the extension, the mesh over the vacuum region is resampled along $s$ to ensure that the spacing in the extended volume remains the same as that used for the plasma region, $s_{v} \leq 1$.

The method just described is the one used for all calculations included in this paper and it shows to work very well. However, it is possible to do the extension in other ways. For instance, a method based instead on using analytical interpolation formulas is described in Appendix A.

\section{B. Magnetic field guess in the extended domain}

When it comes to the construction of an initial guess for the magnetic field in the extended computational volume, several options are possible. Although theoretically equivalent, it turns out that not all of them are equally optimal after being discretized on SIESTA's numerical mesh. To choose among these options, we have required that two conditions be met in order to facilitate the convergence of SIESTA and make the quality of the final solution better (i.e., a smaller force residual). First, the magnetic field must remain smooth over the extended volume to avoid the introduction of spurious eigenvalues in the Hessian of the problem. Secondly, it must have (almost) zero divergence, since SIESTA does not incorporate any divergence-cleaning strategy at this time.

The first way that comes to mind in order to construct a global guess for the magnetic field is probably to use the VMEC solution (that is, the contravariant $B^{\theta_{v}}$ and $B^{\phi_{v}}$ components, since $B^{s_{v}}=0$ ) for all interior isosurfaces (i.e., for $s_{v} \leq 1$ ). In addition, one needs to estimate the three contravariant components of the magnetic field in the vacuum region of the extended coordinate system. We have tested several ways to do this. The first method we have tried is to evaluate Biot-Savart's law at each mesh point with $s_{v}>1$ using the currents that VMEC provides,

$\mathbf{B}^{\mathrm{J}^{\mathrm{VMEC}}}\left(\mathbf{r}, s_{v} \geq 1\right)=\frac{\mu_{0}}{4 \pi} \iiint_{s_{v} \leq 1} d V^{\prime} \frac{\mathbf{J}^{\mathrm{VMEC}}\left(\mathbf{r}^{\prime}\right) \times\left(\mathbf{r}-\mathbf{r}^{\prime}\right)}{\left|\mathbf{r}-\mathbf{r}^{\prime}\right|^{3}}$,

and add to it the vacuum magnetic field created, at the same location, by the external coils [For instance, in the case of W7-X discussed in this paper, the vacuum field has been computed using IPP's MAG3D code ${ }^{13}$.] It is worth pointing out that, although the integration could be carried out directly in SIESTA coordinates, it is more computationally efficient to consider a local cylindrical or cartesian basis for the current vector, and then to project the result of the integral onto the local contravariant basis
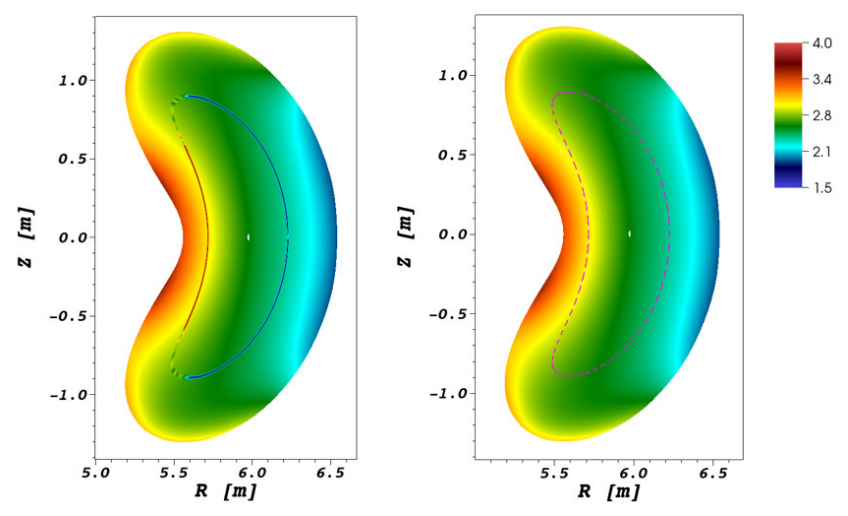

Figure 4. Left: Color plot for $|\mathbf{B}|$ for the W7-X magnetic field obtained by combining the VMEC field for $s_{v}<1$ with the vacuum field plus the one obtained from Biot-Savart's law (Eq. 11) for $s_{v}>$ 1. Right: Color plot for $|\mathbf{B}|$ for the field obtained by combining the vacuum field and the one obtained by integrating Biot-Savart's law using VMEC currents over the whole domain (the $s_{v}=1$ surface is shown in dashed magenta line). Both are shown at the toroidal plane $\phi_{v}=0$.

at $\mathbf{r}$ to get each of the contravariant components of $\mathrm{B}$. Otherwise, Christoffel symbols ${ }^{15}$ must be computed to relate the coordinate basis vectors at any arbitrary pair of locations within the volume, which is a very intensive computation.

A second (and faster) approach is to take advantage of the so-called virtual casing principle ${ }^{16,17}$, that permits to substitute the volume integral in Eq. 11 by the surface integral

$$
\mathbf{B}^{\mathrm{J}^{\mathrm{VMEC}}}\left(\mathbf{r}, s_{v} \geq 1\right)=\frac{\mu_{0}}{4 \pi} \iint_{s_{v}=1} d S^{\prime} \frac{\mathbf{K}\left(\mathbf{r}^{\prime}\right) \times\left(\mathbf{r}-\mathbf{r}^{\prime}\right)}{\left|\mathbf{r}-\mathbf{r}^{\prime}\right|^{3}}
$$

where the current sheet is given by,

$$
\mathbf{K}\left(\mathbf{r}, s_{v}=1\right)=\frac{\left[\mathbf{B}_{\mathrm{VMEC}} \times \mathbf{n}\right]_{s_{v}=1}}{\mu_{0}},
$$

where $\mathbf{n}$ is the (outwards) normal vector at each location in $s_{v}=1$.

We have tested both approaches and found that both of them fail to provide a sufficiently smooth magnetic field across $s_{v}=1$, which messes up SIESTA's convergence (see Fig. 4; left frame, that shows a color plot of $|\mathbf{B}|$ with a clear discontinuity at $s=1$ ). This situation remains, although somewhat improved, even after applying some of the schemes proposed in the literature to remove this discontinuity ${ }^{18}$ (namely, to use again the virtual casing principle to remove the vacuum field contribution from the VMEC solution and to replace it by the vacuum field computed directly from the coils).

It is clear that the easiest way to avoid these discontinuities at $s_{v}=1$ is to avoid any patching at $s_{v}=1$ of fields computed in different ways. For instance, one could discard the VMEC magnetic field for $s_{v} \leq 1$, integrate instead Eq. 11 inside of the plasma region and add 

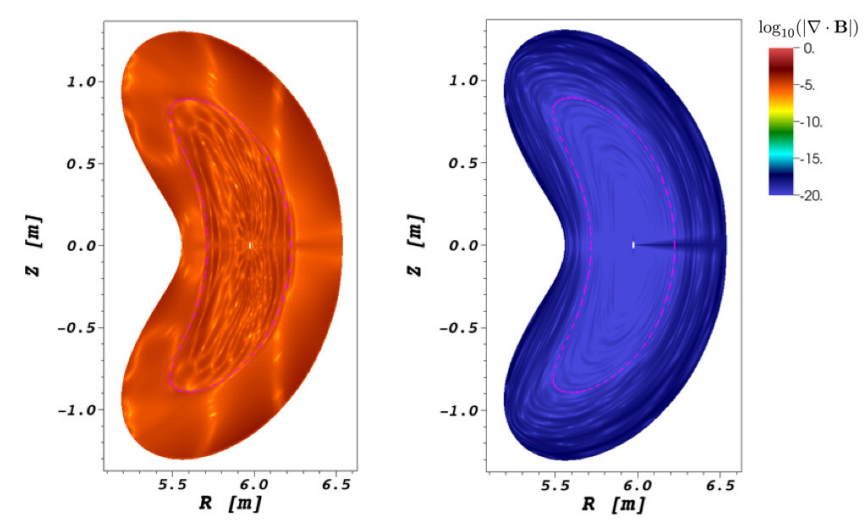

Figure 5. Left: Color plot for $\log (|\nabla \cdot \mathbf{B}|)$ for the W7-X magnetic field obtained by combining the vacuum field and the one obtained from Biot-Savart's law (Eq. 11) over the whole extended domain. Right: Color plot for $\log (|\nabla \cdot \mathbf{B}|)$ for the magnetic field obtained as the curl of the magnetic potential obtained by combining the vacuum one and the one obtained by integrating Eq. 17 (the $s_{v}=1$ surface is shown in dashed magenta line). Both are shown at the toroidal plane $\phi_{v}=0$.

to it the vacuum field generated by the external coils. The resulting field does not have any discontinuity at $s_{v}=1$ (see Fig. 4; right frame). In fact, it often provides a much closer guess to the final solution since it may already contain magnetic islands and stochastic regions within $s_{v}<1$. However, this method is still not an optimal choice since, due to the inaccuracies of the numerical integration of Biot-Savart's law, an unacceptably large non-zero divergence is present for the desired resolution (see Fig. 5; left frame). This excessively large value of the divergence would regretfully be preserved during the iterative procedure, since SIESTA does not perform any divergence cleaning procedure, which would yield a low quality magnetic field after converging.

The divergence problem can be easily resolved by considering instead the magnetic vector potential, $\mathbf{A}=$ $\left(A_{s_{v}}, A_{\theta_{v}}, A_{\phi_{v}}\right)$, and by differentiating it numerically in such a way that the divergence is identically zero on the SIESTA mesh. Indeed, in general coordinates, the magnetic field is obtained as [here, we use the notation $\left.\tilde{B}^{\alpha}=\sqrt{g} B^{\alpha}\right]$ :

$$
\begin{aligned}
\tilde{B}^{s_{v}} & =\frac{\partial A_{\phi_{v}}}{\partial \theta_{v}}-\frac{\partial A_{\theta_{v}}}{\partial \phi_{v}} \\
\tilde{B}^{\theta_{v}} & =\frac{\partial A_{s_{s}}}{\partial \phi_{v}}-\frac{\partial A_{\phi_{v}}}{\partial s_{v}} \\
\tilde{B}^{\phi_{v}} & =\frac{\partial A_{\theta_{v}}}{\partial s_{v}}-\frac{\partial A_{s_{v}}}{\partial \theta_{v}} .
\end{aligned}
$$

Since SIESTA needs the magnetic field on the radial half mesh, defined as, $s_{k}^{h}=d s(k-1 / 2), k=1,2, \cdots N_{s}+$ $N_{s}^{\prime}-1, A_{s_{v}}$ must be defined on the half radial mesh, but $A_{\theta_{v}}$ and $A_{\phi_{v}}$ must both be given on the full radial mesh, $s_{k}^{f}=d s(k-1), k=1,2, \cdots N_{s}+N_{s}^{\prime}$. It is straightforward

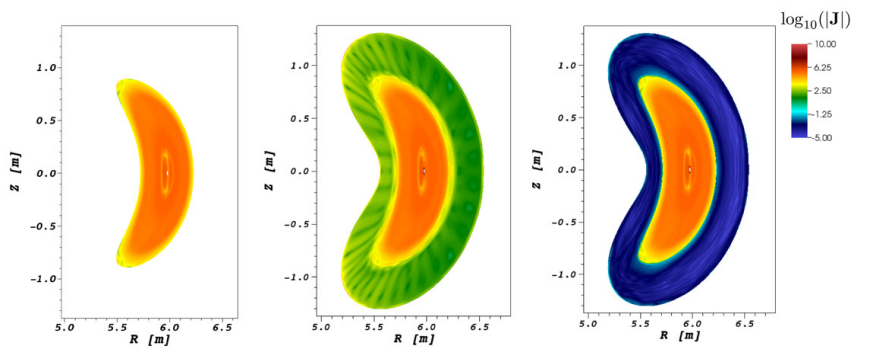

Figure 6. Left: Color plot of $\log (|\mathbf{J}|)$ for the original VMEC solution inside $s_{v}=1$; Middle: color plot for the same quantity as obtained by performing the consistency check described in the text (i.e., evaluating $\mu_{0}^{-1} \nabla \times \nabla \times \mathbf{A}$ ) on the W7-X magnetic potential vector obtained by performing the integral that appears in Eq. 16. Right: Color plot of $\log (|\mathbf{J}|)$ obtained instead by performing the same consistency check on the magnetic potential vector obtained from solving the differential equation Eq. 17. Both are shown at the toroidal plane $\phi_{v}=0$.

to prove that the divergence of the resulting magnetic field now identically vanishes on the half radial mesh.

In order to estimate the vector magnetic potential, we first tested a scheme analogous to the one we outlined earlier for the magnetic field. That is, to integrate numerically

$$
\mathbf{A}^{\mathrm{J}^{\mathrm{VMEC}}}(\mathbf{r})=\frac{\mu_{0}}{4 \pi} \iiint_{s \leq 1} d V^{\prime} \frac{\mathbf{J}^{\mathrm{VMEC}}\left(\mathbf{r}^{\prime}\right)}{\left|\mathbf{r}-\mathbf{r}^{\prime}\right|}
$$

over the whole extended volume, and to add to the result the magnetic potential vector created by the currents flowing in the external coils [provided again by IPP's MAG3D code]. This approach might appear the perfect solution since no patching of solutions is done, thus avoiding all discontinuities at $s_{v}=1$, and a zero divergence of the field is guaranteed over the whole volume. Regretfully, we found that yet another problem appears due to the numerical inaccuracies accumulated during the several coordinate transformations needed to obtain the results on the SIESTA mesh (the integration of Eq. 16 is carried out in Cartesian (or cylindrical) coordinates, and then transformed to SIESTA's coordinates to avoid an expensive evaluation of Christoffel symbols; also, the vacuum magnetic potential is provided by MAG3D in cylindrical coordinates). The problem can be made apparent by performing the consistency check of calculating $\nabla \times(\nabla \times \mathbf{A})\left[=\mu_{0} \mathbf{J}\right]$ on the obtained magnetic vector potential. Clearly, the result should be very close to the original VMEC currents (shown in the left frame of Fig. 6) only for $s_{v}<1$, where the plasma is present, and zero (or very small) everywhere else in the extended computational domain. However, unphysical current densities do appear in the vacuum region, that can locally be significant (see middle frame of Fig. 6).

Although these currents could probably be avoided by carrying out all numerical integrations directly on the SIESTA mesh, the computational cost of doing it this 
way is very large, due to the aforementioned need to evaluate all Christoffel tensors at every point in the mesh. Thus, we have tested another method, that has turned out to be much faster and more efficient. We simply invert numerically Ampére's law inside the extended domain:

$$
\nabla \times\left(\nabla \times \mathbf{A}^{\text {guess }}\right)=\mu_{0} \mathbf{J}^{\mathrm{VMEC}}, \quad s \leq s_{w},
$$

differentiated on the SIESTA extended coordinate system, and using the currents VMEC provides as the source. The procedure is much simpler than any of the ones described earlier, since the $\mathbf{A}^{\text {guess }}$ and $\mathbf{J}^{\mathrm{VMEC}}$ vectors are now related locally, meaning that one does not have to deal with coordinate transformations or Christoffel symbols. To have a unique, meaningful solution, Eq. 17 is supplemented with two boundary conditions that prescribe the value of the vector potential at the $s_{v}=\epsilon \ll 1$ and $s_{v}=s_{w}$ isosurfaces. These values are computed by integrating Eq. 16 and by adding to the result the vacuum potential vector (provided by IPP's MAG3D in this case) at those two surfaces. In this way, the presence of the external coils is felt in the solution through the boundary condition, without having to carry out any additional coordinate transformation. The resulting Poincaré plot for the magnetic field is virtually identical to the one that would be obtained from the integrating procedure but, if the same consistency check is now applied, one finds that all current densities in the vacuum region are virtually zero, as they should be (see Fig. 6, right frame).

After all these trials and tests, the procedure we have finally implemented in SIESTA is to obtain the magnetic potential from Eq.17, and then build the initial guess for the magnetic field evaluating

$$
\mathbf{B}^{\text {guess }}=\nabla \times \mathbf{A}^{\text {guess }}, \quad s_{v} \leq s_{w},
$$

at every point in the SIESTA mesh by using the internal SIESTA's discretization scheme (Eq.13).

\section{Plasma pressure guess in the extended domain}

Regarding the guess for the pressure field, $p^{\text {guess }}(\mathbf{r})$, a natural choice would be to use the pressure profile provided by VMEC for $s_{v}<1$, and to set it to zero elsewhere. This choice leads however to numerical problems for SIESTA's iterative scheme for at least the following reason. The local deviations in pressure and magnetic field that, according to ideal $\mathrm{MHD}^{8}$, a plasma displacement $\xi$ causes from its current state $\left(p_{0}, \mathbf{B}_{0}\right)$ are:

$$
\begin{aligned}
\delta p & =-(\xi \cdot \nabla) p_{0}-\Gamma p_{0} \nabla \cdot \xi \\
\delta \mathbf{B} & =\nabla \times\left(\xi \times \mathbf{B}_{0}\right)
\end{aligned}
$$

If $p_{0}$ vanishes for $s_{v}>1$, it is clear that one could build many different non-zero displacement fields $\xi$ that vanish for $s_{v} \leq 1$, but that are non-zero and parallel to $\mathbf{B}_{0}$ for $s_{v}>1$. None of these displacements change the total MHD energy, since $\delta p=\delta \mathbf{B}=0$ everywhere. Thus, if such displacements are allowed, the kernel of the numerical Hessian of the problem becomes huge which causes severe convergence problems. In order to avoid the formation of such a kernel, we have considered instead a finite, fast-decaying pressure profile for $s_{v}>1$.

\section{Boundary conditions at $s_{v}=s_{w}$}

The last piece needed to complete the free-plasmaboundary extension of SIESTA is to choose the boundary conditions that will be applied at the new fixed boundary, $s_{v}=s_{w}$. In the original SIESTA implementation, $s_{v}=1$ was assumed to stay a true magnetic surface. Thus, it was natural to prescribe $p(1)=B^{s}(1)=0$. In the extended version, this ceases to be the case since $s=s_{w}$ needs not be a magnetic surface [Indeed, the vacuum magnetic field calculated from the coils is usually not tangent to the vacuum vessel.]. Instead, we do impose $p\left(s_{w}\right)=0$ and keep $\mathbf{B}\left(s_{w}\right)$ at the same value that the initial guess for the magnetic field had at $s=s_{w}$. The physical justification for this choice comes from the fact that most vacuum vessels are made of steel (with a conductivity $\left.\sigma \sim 10^{6}(\Omega \cdot m)^{-1}\right)$ and have widths $\Delta \sim(1-2) \mathrm{cm}$, which yields a penetration time for the magnetic field through the vessel of $\tau_{m} \sim \mu \sigma \Delta^{2} \sim(200-300) \mu \mathrm{s}$. Therefore, the vacuum field has plenty of time to penetrate the vessel before the plasma discharge even starts, at least for stellarators. The non-linear response of the plasma is not included in our chosen boundary condition for $\mathbf{B}$, but we think that the error made will be relatively small considering that $s_{w}$ is far from the plasma edge. We will provide numerical evidence supporting this claim for the W7-X case used to illustrate SIESTA's new capabilities in the next section. It is left to a future extension of this work to iterate on this boundary condition by including the changes of the plasma response during the SIESTA iteration.

\section{FREE-PLASMA-BOUNDARY SIESTA CALCULATIONS FOR THE W7-X STELLARATOR}

The Wendelstein 7-X (W7-X) stellarator $^{14}$ is an experimental device located in Greifswald, Germany by the Max-Planck-Institute for Plasma Physics. It is one of the largest stellarators in operation with a major radius $R_{0}=5.5 \mathrm{~m}$, a minor radius $a=0.53 \mathrm{~m}$, and a magnetic field up to $B_{0}=3 \mathrm{~T}$ made possible by superconducting coils. The five period $\left(N_{p}=5\right) \mathrm{W} 7-\mathrm{X}$ has been designed to have low magnetic shear, optimized for small bootstrap current and equipped with an island divertor for particle and power control.

The W7-X configuration that we have chosen to illustrate the new free-plasma-boundary capabilities of 


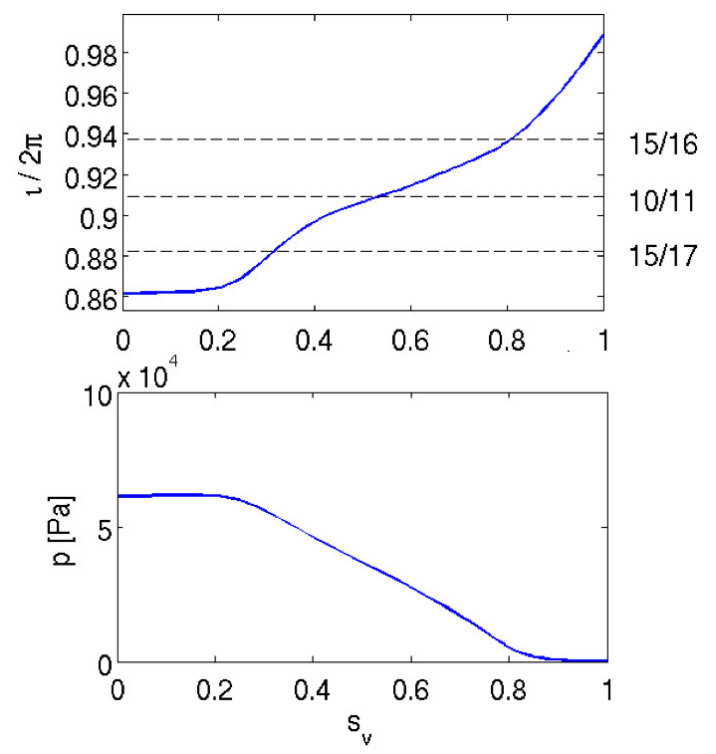

Figure 7. Rotational transform (above) and plasma pressure (below) radial profiles for the W7-X configuration examined in this paper. The locations of the lowest order rational surfaces are indicated, in the rotational transform profile, with horizontal dashed lines.

SIESTA has the rotational transform profile shown in the left frame of Fig. 7. Several rational surfaces exist at locations with $s_{v}<1$ as shown in the figure, but they are of relatively high order. Its more salient feature, however, is that the low-order $5 / 5$ rational is located just outside the plasma edge in order to provide the basis for an island divertor magnetic field topology to isolate the plasma from the vacuum vessel.

The vacuum field for this configuration, as provided by IPP's MAG3D code is shown in Fig. 8. It clearly shows the $5 / 5$ magnetic island just outside the plasma edge considered by VMEC at $s_{v}=1$, whose boundary is shown in magenta. Its presence makes this configuration a particularly useful test case to illustrate the new freeplasma-boundary capabilities of SIESTA, since we expect the island to modify the plasma edge significantly, once the equilibrium solution is extended all the way to the vacuum vessel.

The VMEC equilibrium solution (converged down to a normalized force residual $\left\langle|\mathbf{F}|^{2}\right\rangle \sim 10^{-20}$, where the brackets stand for volume average) naturally has perfectly nested magnetic surfaces all the way to $s_{v}=1$, as advertised (see Fig. 9). We have used $N_{s}=49$ radial points, $M=20$ and $N=16$, that amounts to roughly 700 different harmonics. Cross-sections of the magnetic surfaces obtained by VMEC, for an average $\beta \equiv\left\langle 2 \mu_{0} p / B^{2}\right\rangle \sim 0.8 \%$, are shown in Fig. 9.

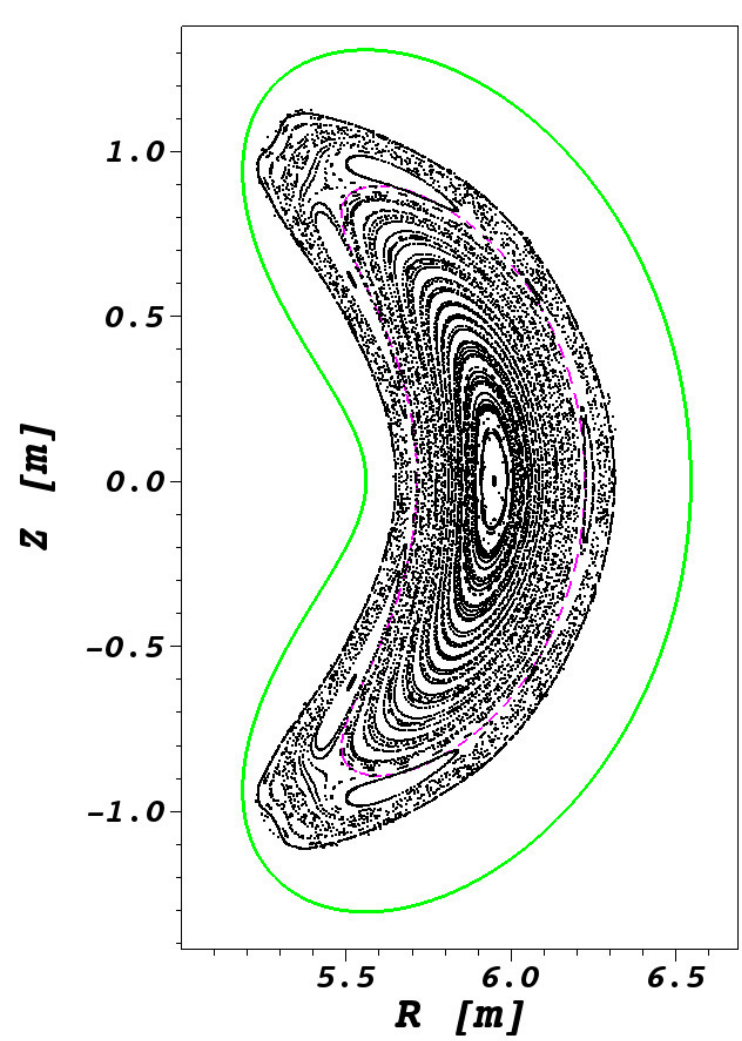

Figure 8. Poincaré plot at the toroidal plane $\phi_{v}=0$ of the vacuum magnetic field for the W7-X configuration used, as provided by IPP's MAG3D code.

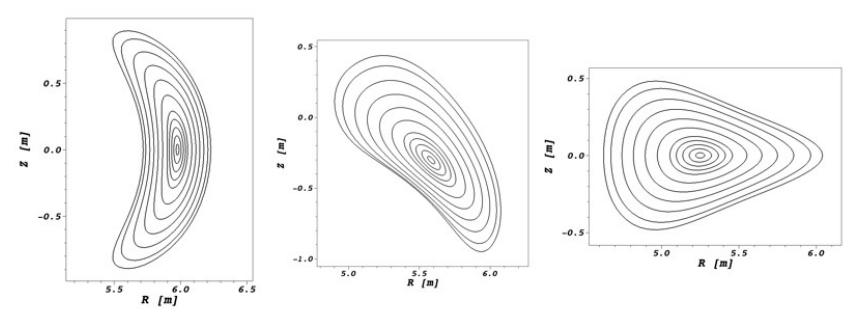

Figure 9. Several toroidal cross-sections (at toroidal angles $\left.\phi_{v}=0, \pi /\left(2 N_{p}\right), \pi / N_{p}\right)$ of the magnetic surfaces obtained by the VMEC code for the W7-X configuration under study.

\section{A. Fixed-boundary calculation}

First, we proceed to run SIESTA in its standard fixedboundary mode. The $\beta \sim 0.8 \%$ VMEC equilibrium previously described provides both the fixed background coordinate system and the seed magnetic field to start the iteration. The number of isosurfaces considered in the SIESTA run is the same as in the VMEC solution, $N_{s}=49$, although the fields have been resampled on SIESTA's radial coordinate, since $s_{v}=\sqrt{s}$. The harmonic content of the solution is also the same as that of 


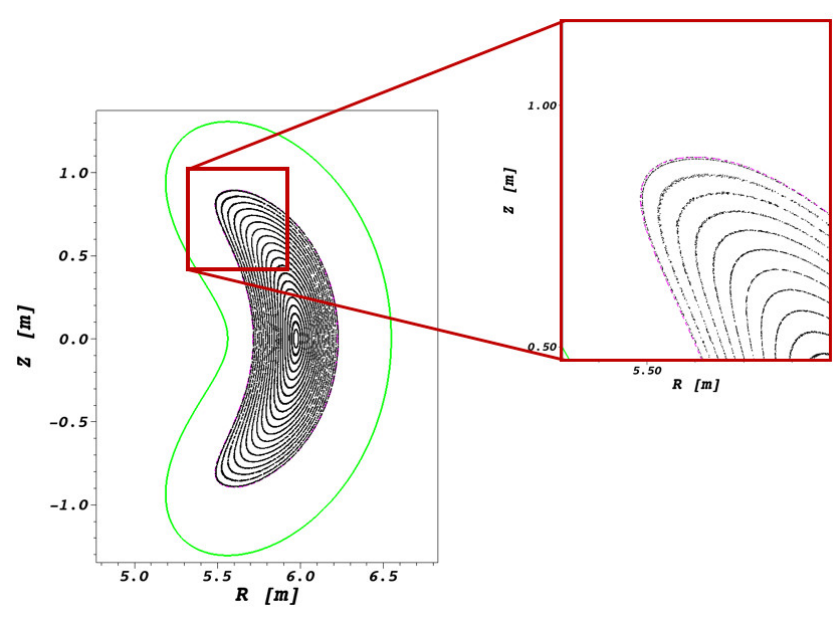

Figure 10. Poincaré plot at the toroidal plane $\phi_{v}=0$ of the magnetic field of the converged equilibrium obtained by SIESTA when run in fixed boundary mode.

VMEC, $M=20$ and $N=16$.

After a few iterations in which a finite, albeit small resistivity is applied, the resistive step is removed and the solution is allowed to converge to a final equilibrium in which the average force residual is of the order of $\left\langle|\mathbf{F}|^{2}\right\rangle \sim 10^{-20}$. No perturbation of the seed field has been applied, but a small, finite resistivity is allowed in the first few iterations to permit the adjustment of the pressure if needed. The final equilibrium magnetic field is shown in Fig. 10, where a Poincaré plot for the final magnetic field is shown at the toroidal cross-section $\phi_{v}=0$. As expected, no visible magnetic island appears for $s_{v} \leq 1$ since no low-order rational surfaces are present there. In fact, it is very similar to the VMEC solution (Fig. 9). The corresponding reduction in the total MHD energy is small with respect to the VMEC solution being roughly $\delta W / W \sim 10^{-7}$.

\section{B. Free-plasma-boundary calculation}

We illustrate next the capabilities of the new extension of SIESTA. As previously advertised, the vacuum vessel of W7-X (or, more precisely, a smoothed-out version of it) is used as the new external boundary. It is shown in green in Fig. 11 (a 3D portion of it is also shown in Fig. 1). The extended mesh that results from following the procedure described in Sec. II A is also shown in Fig. 11. It has a total of 82 isosurfaces (i.e., $N_{s}=49 ; N_{s}^{\prime}=33$ ). The first 49 isosurfaces (that is, those with $s_{v} \leq 1$ ) are in essence those of the VMEC solution, albeit resampled to be evenly spaced with respect to the new radial label $s_{v}=\sqrt{s}$. The next 33 isosurfaces correspond to values of the radial coordinate $s_{v}>1$. The last isosurface, $s_{v}=s_{w}$, naturally coincides with the smoothed-out vacuum vessel. Regarding the number of harmonics, $M=20$ and $N=16$ has been chosen in order to properly capture the complex harmonic content of the external region, introduced in part by the shape of the vacuum vessel. In fact, that is why we chose those values for the previous VMEC runs, in spite of the fact that a good VMEC solution is possible with less harmonics. All the required metric information (the jacobian, for instance, is shown in Fig. 2) has been computed, over the extended mesh, using the procedure described in detail in Sec. II A.

The converged results of the SIESTA free plasma boundary run, which took close to 4 hours running in 16 XEON processors of the E5 family, (with a normalized residual force $\left\langle F^{2}\right\rangle \sim 10^{-18}$ ) are shown in Figs. 12 and 13. The corresponding reduction in the total MHD energy is now significant, $\delta W / W \sim 10^{-5}$, since it is two orders of magnitude greater than the change of energy achieved in the previous fixed-boundary run, where no islands appeared in the equilibrium. Fig. 12 shows the Poincaré plot of the converged magnetic field for the configuration under study. As it is clearly shown, the magnetic island associated to the $5 / 5$ rational surface is now wide enough as to penetrate the $s_{v}=1$ surface (shown in magenta), thus deforming the plasma boundary (see also Fig. 14). This is a direct consequence of the finite pressure of the equilibrium, as it becomes apparent after comparing this Poincaré plot with that shown in Fig. 8 for the vacuum case. The phase of the island in the finite pressure case remains the same as that of the vacuum, in contrast to what is sometimes found for higher- $\beta$ cases, where the phase of the island may also shift. Due to the small $\beta$, the position of the magnetic axis also remains unchanged with respect to the vacuum case, due to the negligible Shafranov shift.

Fig. 13, on the other hand, shows a set of selected isosurfaces for the plasma pressure for two of the W7X
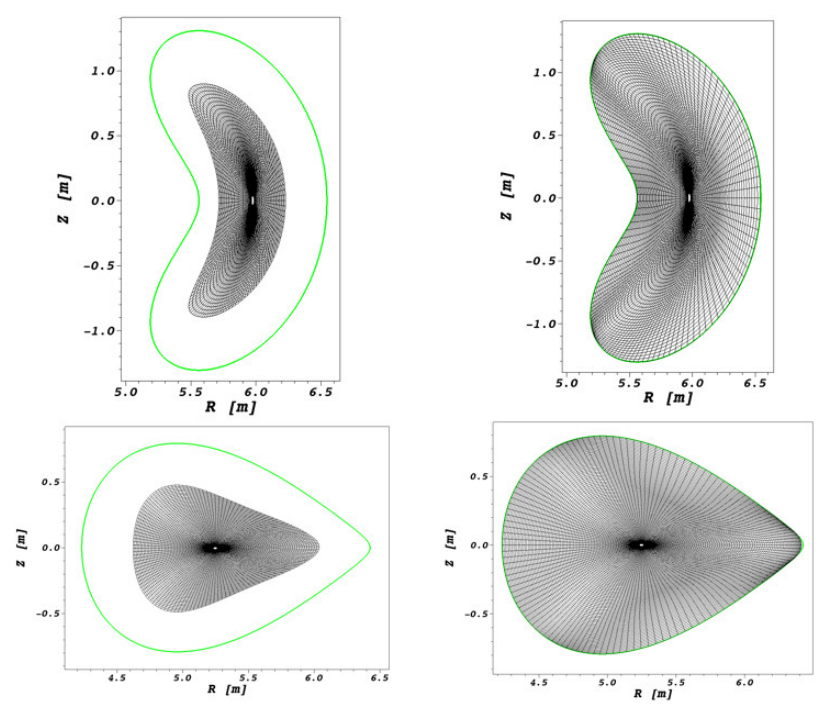

Figure 11. Left: cross-sections of magnetic surfaces at toroidal angles $\phi_{v}=0, \pi / N_{p}$ for $s_{v} \leq 1$, including the vacuum vessel (in green) for W7-X; Right: same cross-sections for the final extended mesh up to $s_{v}=s_{w}$. 


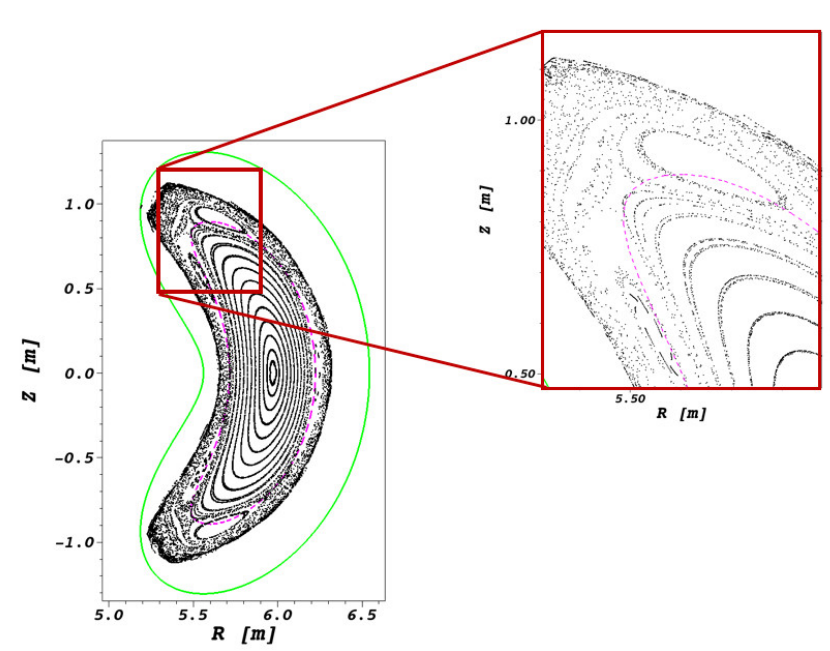

Figure 12. Poincaré at the toroidal plane $\phi_{v}=0$ of the magnetic field of the converged equilibrium obtained by SIESTA when run in free-plasma-boundary mode.
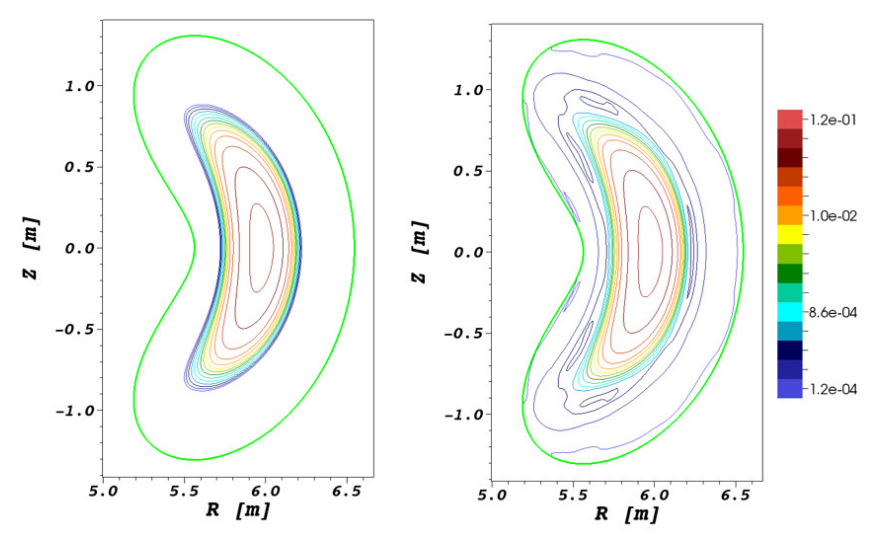

Figure 13. Left: pressure isosurfaces shown at the toroidal section $\phi_{v}=0$ for the equilibrium solution obtained by SIESTA in fixed-boundary for the W7-X equilibrium with $0.8 \%$ discussed in the text; Right: pressure isosurfaces for the converged equilibrium solution found by SIESTA when run in free-plasma-boundary mode instead. In all plots, the $s_{v}=1$ surface is shown in magenta; the $s_{v}=s_{w}$ surface is shown in green.

runs previously described. On the left, contours are shown for the SIESTA fixed-boundary converged solution which, in essence, is identical to the original VMEC solution for this case. On the right, the pressure contours shown correspond to the final equilibrium solution found by SIESTA when run in free-plasma-boundary mode. As can be seen, the few resistive iterations that took place at the beginning of SIESTA's nonlinear iteration have allowed the pressure contours to align themselves with the modified magnetic structure, in order to satisfy $\mathbf{B} \cdot \nabla p=0$. In the process, the shape of the plasma boundary has been modified to adapt to the new topology existent across the region where the $5 / 5$ islands are present, as shown in more detail in Fig. 14. It is also worth noting that the pressure isosurfaces that appear for $s_{v}>1$ correspond to the (very tenuous) pressure that was included in the vacuum region (see discussion in Sec. IIC) in order to avoid a Hessian with a large, nontrival in the linear problem that SIESTA solves at each step of the nonlinear Newton iteration. They are absent in the fixed-boundary solution.

Finally, we are now in the position to check the validity of the assumption made in Sec. IID regarding the modification of the value of the magnetic potential vector at $s=s_{w}$ by the nonlinear plasma response being small. Fig. 15 shows the isolevels of the relative difference at $s_{v}=s_{w}$ between the magnitude of two magnetic vector potentials: the one used as boundary condition, and the one resulting from integrating plasma and coil currents from the converged SIESTA solution. As can be seen, the modification is small as expected, having an average value of just $2 \%$. Furthermore, if we examine only the most important resonant harmonic in this configuration, i.e. $(m=5, n=5)$, the error drops to $0.02 \%$ at the vacuum vessel.

\section{CONCLUSIONS}

A free-plasma-boundary extension of the SIESTA MHD equilibrium code has been presented. By enlarging SIESTA's computational volume, the plasma edge ceases to be considered a fixed boundary, being now able to freely change in order to balance any pressure or magnetic forces that might be present. As a result, SIESTA is now in the position to address problems in which a per-

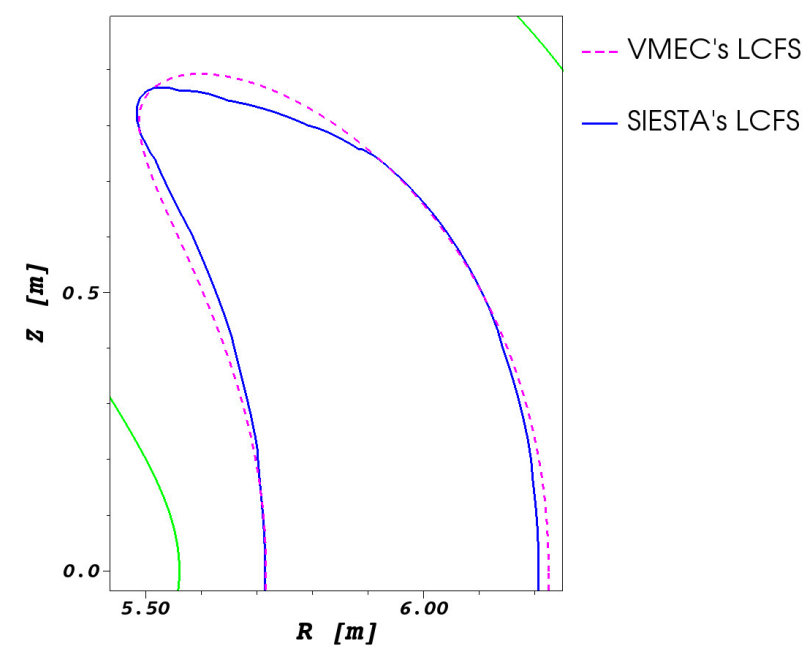

Figure 14. Plasma boundaries at the upper part of the $\phi_{v}=0$ cross-section for the original VMEC and SIESTA fixed-boundary runs (shown in dashed magenta) and the final plasma boundary obtained by SIESTA when run in freeplasma-boundary mode (in blue). 


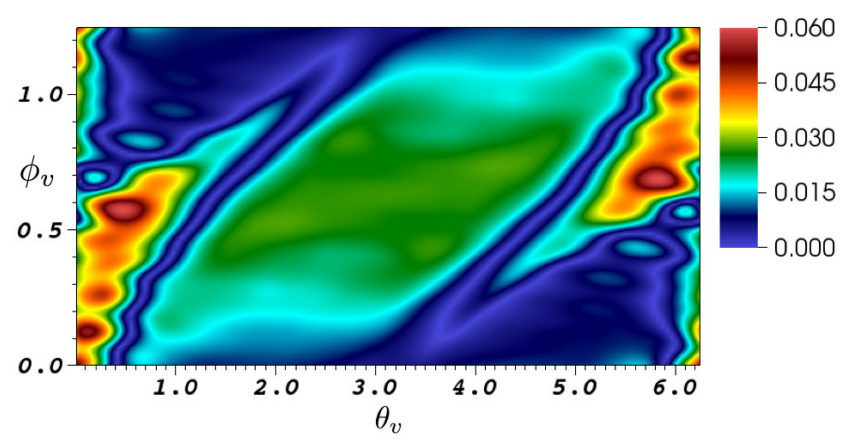

Figure 15. Isolevels of $\left|\mathbf{A}_{1}-\mathbf{A}_{2}\right| /\left|\mathbf{A}_{1}\right|$ on one period of the boundary surface at $s_{v}=s_{w}$. Here, $\mathbf{A}_{1}$ is the value of the magnetic potential vector used as boundary condition (see discussion in Sec. II D) while $\mathbf{A}_{2}$ is the magnetic potential vector obtained by integrating all the currents (plasma and coils) of the final SIESTA solution.

turbed plasma edge might become important, such as the application of edge magnetic resonant perturbations ${ }^{12}$, or the possible distortion of the plasma edge due to unbalanced plasma currents such as bootstrap or heatinginduced currents ${ }^{19}$.

It is important to keep in mind that, in all SIESTA free-plasma-boundary runs, the vacuum magnetic field (or, more precisely, the magnetic vector potential) created by the external coils must be explicitly provided. At least, on the two surfaces used to provide the boundary conditions to solve Eq. 17. For the W7-X configuration studied in this paper, the vacuum vector potential has been calculated by means of IPP's MAG3D code. But for applications to other devices, this information will have to be provided by the user. Work is currently underway to make SIESTA capable of extracting this information from the same files (the so-called (mgrid) files) that VMEC uses when run in free-boundary mode ${ }^{20}$.

\section{APPENDIX A. ALTERNATIVE MESH EXTENSION SCHEME VIA INTERPOLATION FORMULAS.}

The mesh extension procedure that was described in Sec. II A relied on the building of a proper database of points over the extended domain, from which the $s_{v^{-}}$ isosurfaces of the SIESTA coordinate systems was built. The starting point was a set of intersections of the extrapolated poloidal rays of the VMEC coordinate system with the last closed surface (usually the vacuum vessel), calculated at each toroidal plane. The undesired accumulation of these intersection points that often appears near regions with large indentation was dealt with by point resampling over the last closed surface, if needed. This method works very well and has been used in all the calculations included in the paper, but it is not the only one possible.

In this appendix, we discuss a second possibility based
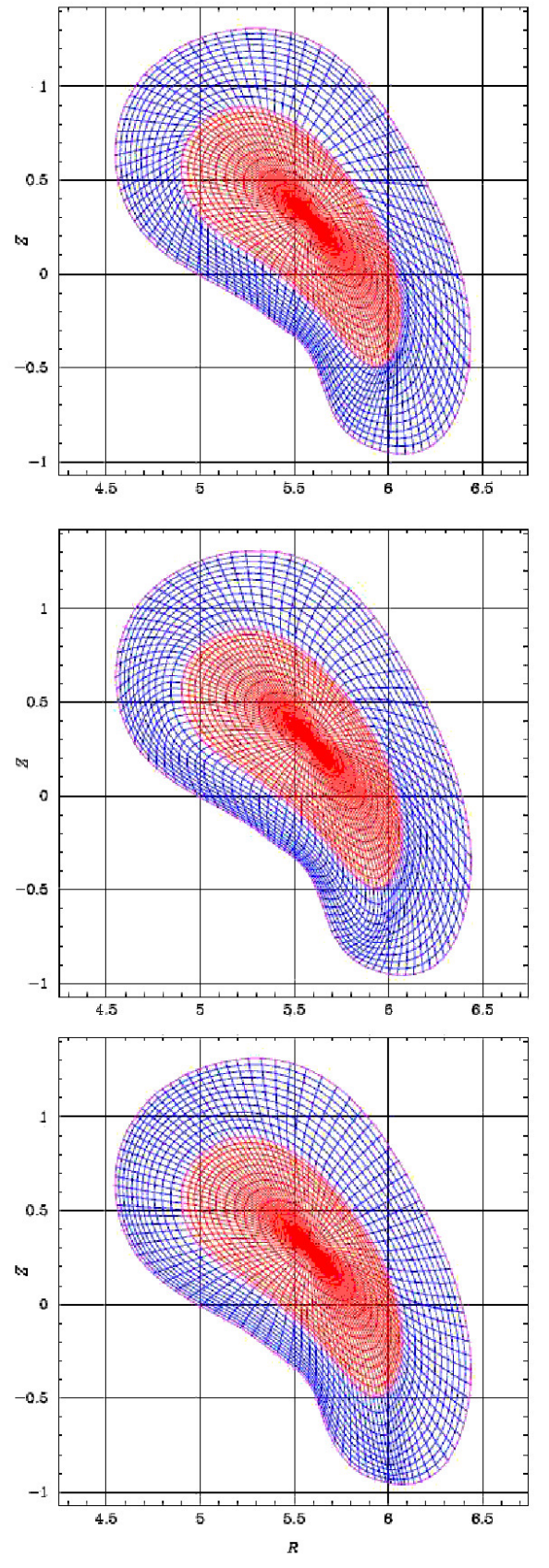

Figure 16. Cross sections at toroidal angle $\phi=\pi /\left(2 N_{p}\right)$ of the magnetic surfaces obtained over the extended volume (in red, the original VMEC surfaces for $s_{v} \leq 1$; in blue, over the extended region) for the W7-X configuration examined in Sec. III B using linear, quadratic and quadratic with slip interpolation formulas discussed in Appendix A.

on the use of interpolation formulas. The starting point is to express the vacuum vessel position in a Fourier series analogous to the one VMEC uses, although with $\theta$ the 
geometrical angle, not VMEC's internal angle.

$$
\begin{aligned}
R^{\mathrm{VV}}(\theta, \phi) & =\sum_{m, n} R_{m n}^{\mathrm{VV}} \cos \left(m \theta-n N_{p} \phi\right) \\
Z^{\mathrm{VV}}(\theta, \phi) & =\sum_{m, n} R_{m n}^{\mathrm{VV}} \sin \left(m \theta-n N_{p} \phi\right),
\end{aligned}
$$

We will also introduce the notation,

$$
\begin{aligned}
& R^{1}(\theta, \phi)=\sum_{m, n} R_{m n}\left(s_{v}=1\right) \cos \left(m \theta-n N_{p} \phi\right) \\
& Z^{1}(\theta, \phi)=\sum_{m, n} Z_{m n}\left(s_{v}=1\right) \sin \left(m \theta-n N_{p} \phi\right)
\end{aligned}
$$

for the positions of the points located at the last VMEC closed surface, $s_{v}=1$, at the same angle value (i.e., for $\left.\theta^{*}=\theta\right)$.

We now seek an interpolation formula that will generate the extension of the mesh between VMEC's last closed surface and the vacuum vessel while, at the same time, providing continuity of all quantities and their derivatives across the $s_{v}=1$ surface. It turns out that linear interpolation is not good enough. Indeed, if one tries, for fixed $\theta$ and $\phi$ (we drop their dependence in what follows, although it is implicitly assumed), a linear formula such as:

$$
\begin{aligned}
& R(\rho)=R^{1}+\rho\left(R^{\mathrm{VV}}-R^{1}\right) \\
& Z(\rho)=Z^{1}+\rho\left(Z^{\mathrm{VV}}-Z^{1}\right),
\end{aligned}
$$

one obtains an interpolation that has no continuous derivative at $s=1$. This is clearly appreciated in the upper frame of Fig. 16, that shows the cross-section of the extended coordinate system for the W7-X configuration at toroidal angle $\phi=\pi /\left(2 N_{p}\right)$. The reason is that $\theta$ and $\theta^{*}$ are very different angles, although we have used them as if they were the same.

This limitation can be avoided by moving to a quadratic representation such as:

$$
\begin{aligned}
R(\rho)= & \left.\frac{R_{s}^{1}}{2}\left[1-\rho^{2}-(1-\rho)^{2}\right)\right]+R^{1}+ \\
& +\rho^{2}\left(R^{\mathrm{VV}}-R^{1}\right) \\
Z(\rho)= & \left.\frac{Z_{s}^{1}}{2}\left[1-\rho^{2}-(1-\rho)^{2}\right)\right]+Z^{1}+ \\
& +\rho^{2}\left(Z^{\mathrm{VV}}-Z 1\right) .
\end{aligned}
$$

Here $R_{s}^{1}$ and $Z_{s}^{1}$ are the radial derivatives at the $s_{v}=1$ surface. The result of using the second-order interpolation is shown in the middle frame of Fig. 16. Clearly, all derivatives are now continuous across the $s_{v}=1$ surface. However, some additional curvature (to the poloidal rays) has been introduced in the process that would introduce undesired additional angular dependences in the metric tensor, compared to how they behave inside $s_{v}=1$. These undesired effects can be ameliorated by introducing a poloidal slip function $\lambda(\theta)$ that allows each of the initial vessel points to slide poloidally along the vessel while preserving the vessel shape (in a spirit that is very similar to how points on the vessel were poloidally redistributed in the procedure described in Sec. II A to avoid accumulation near regions with large indentation):

$$
\begin{aligned}
& R(\lambda, \theta)=R_{\mathrm{VV}}(\theta+\lambda) \\
& Z(\lambda, \theta)=Z_{\mathrm{VV}}(\theta+\lambda) .
\end{aligned}
$$

The only requirement on $\lambda$ is that $1+d \lambda / d \theta>0$, so that the new angle distribution stays monotonic. We determine $\lambda(\theta)$ by minimising (as a function of $\theta$, at each toroidal plane) the curvature of each poloidal ray, roughly given by:

$$
\kappa^{-1} \sim \sqrt{R_{\rho \rho}^{2}+Z_{\rho \rho}^{2}}
$$

The significant improvement of applying the sliding is illustrated in the lower frame of Fig. 16, where the largely reduced curvature of the poloidal rays for $s_{v}>1$ is apparent.

\section{ACKNOWLEDGEMENTS}

Research funded in part by the Ministerio de Economía, Industria y Competitividad of Spain, grant No. ENE2015-68265. Research carried in part at the Max-Planck-Institute for Plasma Physics in Greifswald (Germany), whose hospitality is gratefully acknowledged. Research supported in part by the US Department of Energy, Office of Fusion Energy Sciences under Award DEAC05-00OR22725. SIESTA runs have been carred out in Uranus, a supercomputer cluster located at Universidad Carlos III de Madrid and funded jointly by European Regional Development Funds (EU-FEDER) project No. UNC313- 4E-2361, and by the Ministerio de Economía, Industria y Competitividad via the National Projects No. ENE2009-12213-C03-03, No. ENE2012-33219, and No. ENE2012-31753.
1 S.P. Hirshman, R. Sanchez and C.R. Cook. "SIESTA: A scalable iterative equilibrium solver for toroidal applications", Phys. Plasmas 18, 062504 (2011).

2 A.H. Reiman and H.J. Greenside. "Computation of zero$\beta$ three-dimensional equilibria with magnetic islands", J.
Comput. Phys. 87, 349 (1990)

3 Y. Suzuki, Y. Nakamura, K. Kondo, T. Hayashi, S. Lloyd and H. Gardner. "MHD equilibrium of a low-shear helical axis Heliotron", Plasma Phys. Contr. Fus. 45, 971 (2003) 
4 S.R. Hudson, R.L. Dewar, G. Dennis, M.J. Hole, M. McGann, G. von Nessi, and S. Lazerson. "Computation of multi-region relaxed magnetohydrodynamic equilibria", Phys. Plasmas 19, 112502 (2012).

5 S.P. Hirshman and J.C. Whitson. "Steepest-descent method for three-dimensional magnetohydrodynamic equilibria", Phys. Fluids 263553 (1983)

6 S.P. Hirshman and H.K Meier. "Optimal Fourier representations for three-dimensional magnetic surfaces", Phys. Fluids 281387 (1985)

7 R.L. Dewar and S.R. Hudson. "Stellarator symmetry", Physica D 112, 275 (1998)

8 J.P. Freidberg. "Ideal magnetohydrodynamics", Plenum, New York (1987)

9 A. Bhattacharjee, T. Hayashi, C.C. Hegna, N. Nakajima and T. Sato. "Theory of pressure-induced islands and self-healing in three-dimensional toroidal magnetohydrodynamic equilibria", Phys. Plasmas 2, 883 (1995).

10 J. Loizu, S. Hudson, A. Bhattarcharjee and P. Helander. "Magnetic islands and singular currents at rational surfaces in three-dimensional magnetohydrodynamic equilibria", Phys. Plasmas 22022501 (2015)

11 J. Loizu, S. Hudson, A. Bhattacharjee and P. Helander. "Magnetic islands and singular currents at rational surfaces in three-dimensional magnetohydrodynamic equilibria", Phys. Plasmas 22022501 (2015)

12 T.E. Evans. "Resonant magnetic perturbations of edgeplasmas in toroidal confinement devices", Plasma Phys.
Contr. Fus. 53, 123001 (2015)

13 J. Svensson, A. Werner. "Large Scale Bayesian Data Analysis for Nuclear Fusion Experiments", 2007 IEEE International Symposium on Intelligent Signal Processing, 1-6 (2007)

14 C. Beidler, G. Grieger, F. Herrnegger, E. Harmeyer, J. Kisslinger, W. Lotz, H Massberg, P. Merkel, J. Nührenberg, F. Rau, J. Sapper, F. Sardel, R. Scardovelli, A. Schlüter and H. Wobig. "Physics and Engineering Design for Wendelstein 7-X", Fusion Technology 17148 (1990)

15 M. Itskov. "Tensor Algebra and Tensor Analysis for Engineers", Springer Verlag, Heidelberg (2015)

16 V.D. Shafranov and L.E. Zakharov. "Use of the virtualcasing principle in calculating the containing magnetic field in toroidal plasma systems", Nucl. Fusion 12599 (1972)

17 J.D. Hanson. "The virtual-casing principle and Helmholtz's theorem", Plasma Phys. Contr. Fus. 57115006 (2015)

18 M. Drevlak, D. Monticello and A. Reiman. "PIES free boundary stellarator equilibria with improved initial conditions", Nucl. Fusion 45731 (2005)

19 J. Geiger, C.D. Beidler, Y. Feng, H. Massberg, N.B. Marushchenko and Y. Turkin. "Physics in the magnetic configuration space of $W^{\gamma}-X^{\prime \prime}$, Plasma Phys. Contr. Fus. 57014004 (2015)

20 S.P. Hirshman, W.I. van Rij and P. Merkel. "Threedimensional free boundary calculations using a spectral Green's function method", Comp. Phys. Comm. 43143 (1986) 